\title{
Civilisations
}

Revue internationale d'anthropologie et de sciences

humaines

39 | 1991

Japon : les enjeux du futur

\section{Crise des stratégies syndicales et évolution de la relation salariale au Japon}

\section{Annie Garanto}

\section{OpenEdition}

\section{Journals}

Édition électronique

URL : http://journals.openedition.org/civilisations/1658

DOI : 10.4000/civilisations. 1658

ISSN : 2032-0442

\section{Éditeur}

Institut de sociologie de l'Université Libre de Bruxelles

\section{Édition imprimée}

Date de publication : 30 octobre 1991

Pagination : 191-248

ISBN : 2-87263-044-9

ISSN : 0009-8140

Référence électronique

Annie Garanto, "Crise des stratégies syndicales et évolution de la relation salariale au Japon », Civilisations [En ligne], 39 | 1991, mis en ligne le 06 juillet 2009, consulté le 02 mai 2019. URL : http:// journals.openedition.org/civilisations/1658; DOI : 10.4000/civilisations.1658 


\title{
CRISE DES STRATEGIES SYNDICALES ET EVOLUTION DE LA RELATION SALARIALE AU JAPON
}

\author{
Annie GARANTO
}

Introduction

Comme dans la plupart des pays européens, le syndicalisme japonais n'échappe pas à la crise. Ses effectifs stagnent et son influence sociale et politique s'affaiblit. Mais au même moment, s'engage un processus de réunification du mouvement syndical qui, par son ampleur, semble ouvrir de nouvelles perspectives.

Comment interpréter ces deux phénomènes qui semblent contradictoires? Ils sont le résultat d'un long processus de crises successives qui remontent pour le Japon au début des années 60 . C'est à cette époque charnière que se produit la principale division du mouvement syndical et que s'élabore les pratiques actuelles des relations du travail. C'est aussi à cette période que la relation salariale se transforme.

Car au-delà des explications conjoncturelles, le mode d'intégration de la main-d'oeuvre au marché du travail semble être le fait central, en renvoyant à la différenciation du monde salarial ${ }^{1}$.

Comprendre les clivages du mouvement syndical, sa volonté de réunification actuelle et ses effets sur la scène politique, c'est aussi analyser les moyens recherchés par les syndicats pour faire face à la réduction de leur audience. Mais c'est d'abord mettre en évidence les causes de ce déclin qui renvoient à la relation salariale. Aussi, nous nous interrogerons sur les crises des stratégies syndicales, en les reliant chaque fois aux transformations de la relation salariale ${ }^{2}$. On tentera de 
dégager alors l'évolution du mouvement syndical et la diversité de ses réponses.

1. Le déclin d'un syndicalisme de revendication et l'apparition d'un système de relations du travail modeme (1960-1970)

Dans le début des années 1960, on assiste à un changement des orientations syndicales : le passage d'un syndicalisme de revendication à un syndicalisme de coopération. Cette évolution s'est opérée au moment de la période de haute croissance où le taux moyen de croissance du PNB était d'environ $10 \%$ par an. Soutenue en grande partie par une accumulation rapide du capital, la croissance a permis de dégager des conditions favorables : le plein emploi puis la hausse des salaires induite par la pénurie de main-d'oeuvre jeune. A cette époque, les grandes entreprises ont consolidé leurs marchés internes du travail, c'est-à-dire maîtrisé davantage la relation salariale des employés permanents. Ces nouvelles stratégies patronales vont profondément bouleverser le paysage syndical.

1.1 La stratégie des employeurs : une politique de maîtrise de la relation salariale

Après les grandes grèves des années 1950 dans les secteurs tels que la sidérurgie ${ }^{3}$ ou les mines ${ }^{4}$ qui avaient pour motif des licenciements collectifs, les employeurs ont changé de stratégie. Pour éviter des conflits longs et coûteux, ils ont recherché une meilleure intégration des salariés à l'entreprise. Cette dernière a trois fondements : une relation salariale spécifiée par un type de promotion, une gestion participative de la main-d'oeuvre et l'intégration sociale des salariés. 
Maîtriser la relation salariale

Les caractéristiques de la relation salariale dans les grandes entreprises, pour les employés permanents ont été très souvent analysées ${ }^{5}$. On en résumera ici les principaux traits :

1.La promotion du salarié (mobilité ascendante) dépend de la qualification fondée sur l'expérience acquise dans l'entreprise. Il y a donc une identification entre qualification et ancienneté (équivalente ici à l'expérience).

2. Le salaire selon le critère de l'ancienneté est la règle qui spécifie cette identification. Il renseigne aussi sur le type de mobilité des salariés. Ceux-ci seront amenés à privilégier la mobilité interne à l'entreprise ${ }^{6}$ par rapport à une mobilité externe (vers le marché du travail).

C'est ce contrôle du système de promotion à l'intérieur de l'entreprise qui a été l'enjeu d'une lutte entre employeurs et syndicats. Le cas de l'industrie sidérurgique est à cet égard exemplaire par l'intensité des luttes qui s'y sont déroulées et la précocité des politiques patronales conduites au milieu des années $1950^{7}$. Dans une période d'expansion et de développement de cette industrie, les employeurs souhaitaient réussir la rationalisation du processus de production. Il s'agissait de répondre à l'accroissement de la demande. Mais à cette période, de nombreux conflits, indices de l'influence des syndicats de revendication, apparaissaient concernant l'organisation du travail et l'élévation de la productivité dans l'atelier (graphe 1).

En effet à cette époque, la production était gérée et s'articulait autour de la qualification du contremaître qui était souvent représentant syndical ${ }^{8}$. L'atelier demeurait donc un lieu important de solidarité et d'organisation des salariés. 
Aussi, les employeurs ont-ils cherché à maîtriser la relation salariale : c'est-à-dire à s'assurer du contrôle de la qualification et de la promotion dans l'entreprise. Pour cela, les employeurs ont introduit des principes classiques de division du travail entre la ligne de production et le bureau. Ces nouvelles méthodes, comme l'étude des temps, ont permis la maîtrise de la qualification dans l'atelier, qui n'était plus laissée au seul contremaître.

En parallèle, une gestion décentralisée du personnel est introduite grâce à la création d'un nouveau type de contremaître (Sagyochosei). Ce dernier est chargé de distribuer les postes en fonction des compétences individuelles, de gérer les augmentations de salaires et les promotions des salariés de l'atelier. Ainsi, le processus d'acquisition de la qualification et donc de la promotion, dépend de lappréciation $\mathrm{du}$ contremaître ${ }^{9}$ et non plus du seul critère de l'âge ou de l'ancienneté de service.

Cette politique patronale a eu un double objectif :

- d'une part, le système de promotion et d'acquisition des qualifications est contrôlé par la direction de l'entreprise;

- de l'autre, l'extension des fonctions de contremaître à la gestion du personnel l'assimile pour certaines entreprises au personnel de direction représentant les actionnaires, l'excluant ainsi de la clause de l'union-shop et donc du syndicat ${ }^{10}$.

La gestion participative des salariés

Enfin, une politique de participation active des salariés est lancée. Cette dernière va prendre sa véritable expansion avec les activités de petits groupes, prolongés ensuite par le mouvement des cercles de qualitélil. Ces nouvelles méthodes répondent au relâchement de l'intégration sociale au niveau de 
l'atelier. Les transformations techniques, comme l'automatisation, entraînent une réduction de la coopération et isolent le salarié. Pour répondre à cette crise et recréer une communauté de travail, ces groupes offrent une structure informelle de mobilisation des salariés ${ }^{12}$. Fondée en principe sur le volontariat, cette structure permet aux salariés d'exprimer leur point de vue pour améliorer la qualité des produits, et rationaliser le processus de production.

Par ailleurs, ces groupes recréent une communauté de travail non plus sur une base syndicale mais sur une structure instaurée par la direction de l'entreprise, qui lui permet d'accroître la communication vers le haut. Il s'agit de gérer les attentes provenant de l'atelier en les orientant vers l'amélioration de la productivité.

L'intégration sociale des salariés

Cette politique plus ancienne, souvent associée à un paternalisme patronal ${ }^{13}$, représente également une autre forme de mobilisation du personnel. Si les prestations sociales sont relativement faibles au Japon ${ }^{14}$, il n'en demeure pas moins qu'il existe un salaire indirect octroyé par l'entreprise. Ce salaire socialisé est constitué par un système d'avantages en nature accordés aux employés permanents de la grande entreprise ${ }^{15}$.

Cette socialisation de la vie privée par l'entreprise permet une gestion de la main-d'oeuvre non seulement dans le travail mais également dans sa vie privée. Le sentiment de loyauté et d'identité à l'entreprise repose donc sur des liens économiques et sociaux très forts. Cependant ce système est limité au noyau dur du salariat : les employés permanents des grandes entreprises, qui constituent également la base militante des syndicats. 
La stratégie d'intégration des salariés à l'entreprise a été menée sur une longue période. Deux objectifs principaux ont été recherchés par les employeurs :

- D'une part, il s'agissait d'accroître la productivité en imposant de nouvelles formes d'organisation du travail ainsi qu'une participation active des salariés. De plus, la relation salariale instituée en encourageant l'acquisition des qualifications accroît en retour la productivité globale de l'entreprise ainsi que ses capacités d'innovation.

- De l'autre, grâce à la maîtrise de la relation salariale, c'est-àdire du système de promotion et de qualification, les employeurs renforçaient l'identification à l'entreprise. Ainsi ont-ils intégré plus fortement la catégorie des contremaîtres et ouvriers qualifiés qui formaient la base des militants syndicaux. Le patronat mobilise en sa faveur cette fraction des salariés, réduisant l'influence des syndicats de revendication. Ceci se traduit d'ailleurs, dès le milieu des années 1960, par une baisse du nombre de conflits ainsi que de jours perdus pour fait de grève (graphe 1).

Confrontés à ces initiatives patronales qui réduisent le champ de l'action revendicative, les syndicats ont dû réviser leurs stratégies.

\subsection{La recomposition du paysage syndical : du syndicalisme de revendication à un syndicalisme de coopération}

Syndicats de revendication, syndicats de coopération

Si l'on effectue une typologie simplifiée des syndicats, on reconnaîtra deux grandes catégories : le syndicalisme de revendication et le syndicalisme de coopération ${ }^{16}$. Caractérisé par une idéologie d'opposition au patronat ${ }^{17}$, le syndicalisme de revendication utilise souvent le moyen de la grève pour 
résoudre les conflits du travail. Il peut aussi bien occuper l'arène économique que politique pour conduire à un changement social plus profond. Certaines fédérations du Sôhyô semblent se rattacher à ce type de syndicalisme, jouant tantôt sur la scène économique, pour des revendications salariales par exemple, tantôt sur la scène politique par son soutien aux partis d'opposition. En revanche, le syndicalisme de coopération présente une idéologie qui souligne la complémentarité des deux acteurs sociaux dans l'entreprise. Cette affirmation de la complémentarité détermine une attitude de coopération ${ }^{18}$ où le règlement des conflits se fait davantage par la négociation informelle. Si le syndicalisme peut agir sur la scène politique, c'est davantage comme un groupe de pression que comme un acteur du changement social. La confédération du Dômei illustre ce deuxième type de syndicat. Quelles sont les raisons du déclin progressif du syndicalisme de revendication, dans la période de la haute croissance ? Comment le mouvement syndical se recompose-t-il ? Telles sont les questions que l'on tentera de cerner.

L'échec des actions revendicatives du milieu des années 1950 est dû en grande partie à la stratégie patronale de maîtrise de la relation salariale ${ }^{19}$. Mais celle-ci s'est acompagnée d'une politique très active d'éviction. En effet, l'implantation d'une gestion participative ne s'est pas faite sans mouvements d'opposition ni de conflits; car ces réorganisations entraînaient une réduction du champ d'action syndical. Il se déroula ainsi une lutte d'influence dont l'enjeu principal était la mobilisation des salariés.

Pour réduire le monopole syndical, les employeurs ont utilisé une stratégie de division par la création de contresyndicats, soit en favorisant des syndicats maisons, soit en opposant les fédérations qui composent le Sôhyô. 
Dans la sidérurgie, la politique de rationalisation (Gorika) menée sur une longue période (1950-1975) aboutit à l'érosion de la puissance syndicale du Tekko Roren. Celui-ci représentait une des fédérations les plus combatives au début du mouvement de "l'offensive du printemps" au sein du Sôhyô. Dès les années 1960, après les échecs successifs des grèves, cette fédération adopte une attitude plus modérée. En 1966, le Tekko Rôren; toujours affilié au Sôhyô, rejoint la fédération internationale des métallurgistes-Comité pour le Japon (IMF-JC) structure souple qui recoupe les confédérations. Fortement orienté vers la coopération, le IMF-JC va jouer également un rôle de modérateur des revendications salariales. Au contraire, pour les secteurs comme les mines ou l'automobile, ces stratégies patronales ont abouti à affaiblir ou faire disparaître les syndicats de revendication ${ }^{20}$. Ces échecs ont d'importantes répercussions : le changement d'orientation du Sôhyô et l'émergence d'une nouvelle confédération : le Dômei.

La réorientation des stratégies du Sohyo

Face aux échecs de sa stratégie, la confédération du Sôhyô, syndicat de revendication, va être confrontée à un double changement. D'une part, la tendance minoritaire modérée du Sôhyô opère une scission pour fonder une nouvelle confédération : le Zenrô Kaigi, prélude à l'émergence du Dômei ${ }^{21}$.

De l'autre, on assiste à une modification de l'orientation du Sôhyô qui conduit à des divergences entre ses fédérations. Jusqu'au début des années 1960, le Sôhyô axait sa politique sur "la lutte dans l'atelier" (Shokuba Tôsô). Cette stratégie cherchait à lutter contre les nouvelles politiques patronales de gestion pour renforcer son pouvoir de négociation concernant les conditions de travail et l'affectation des postes dans 
l'atelier ${ }^{22}$. L'échec de cette stratégie marque l'abandon des revendications concernant l'organisation du travail.

Mais surtout, elle indique que le syndicat de revendication a perdu le contrôle du système de promotion et de qualification dans l'entreprise. Dès lors, son influence vis-à-vis des ouvriers qualifiés se réduit, pour voir peu à peu s'amenuiser sa propre base syndicale dans le secteur privé. Le changement de direction symbolise la nouvelle orientation du Sôhyô ${ }^{23}$.

Sa nouvelle stratégie se concentre davantage sur les gains salariaux. Ainsi le syndicalisme de revendication passe d'une optique de contrôle de la production dans l'entreprise à une optique qui ne s'intéresse qu'à l'aspect de la répartition, c'est-àdire aux négociations salariales. Comme celles-ci ne peuvent acquérir une certaine efficacité qu'au niveau national, l'action du Sôhyô abandonne l'atelier (espace local) pour opérer une centralisation de l'action (espace national).

Cette stratégie est illustrée par l'offensive du printemps (Le Shuntô), lancée en 1955 par le Sôhyô. Ce mouvement concentre les revendications salariales sur une même période et les unifie au niveau national. Il a l'avantage de surmonter la faiblesse des négociations pratiquées au niveau de l'entreprise, pour parvenir à une norme globale de hausse des salaires.

La logique du Shuntô se fonde sur une philosophie du partage équitable des fruits de la croissance. La progression des salaires devait tenir compte des gains de productivité, ainsi que de l'inflation. Les branches motrices, telles que la sidérurgie, diffusaient les hausses salariales dans le reste de l'économie.

Dès 1956, le Shuntô est étendu au secteur public ainsi qu'à d'autres syndicats tels que le Churitsu Rôren. La philosophie du Shuntô a pu relativement bien fonctionner pendant la période 
de haute croissance, où l'on remarque une forte progression des salaires réels (près de $15 \%$ de 1965 à 1973), ainsi qu'une réduction des disparités entre les secteurs et selon les tailles des entreprises (graphe 2). En revanche le dispositif du Shuntô a rétréci le champ des négociations au seul aspect de la répartition : la question salariale. Cette stratégie adoptée par la confédération, laisse au niveau local le champ libre aux fédérations et aux syndicats d'entreprises, qui adoptent des positions divergentes.

Ainsi, dans le secteur privé, certaines fédérations du Sôhyô, comme le Tekko-Roren, abandonne le syndicalisme de revendication, pour adopter une politique de coopération dans l'entreprise. Seuls quelques syndicats d'entreprises minoritaires poursuivent un syndicalisme de renvendication. En revanche, le secteur public dominé par le Sôhyô, semble être le refuge du syndicalisme de revendication. Ainsi, des fédérations, telles que les chemins de fer nationaux, la poste ou les fonctionnaires, ont menée une action sur le terrain visant à contrôler la relation salariale à leur profit ${ }^{24}$. Dans les années 1960, le clivage entre syndicalisme de coopération et syndicalisme de revendication traverse aussi la confédération du Sôhyô. Celle-ci sortira affaiblie par la lutte interne que mènent ses fédérations, pour le contrôle de la direction.

\section{La recomposition du paysage syndical}

Enfin, la deuxième répercussion de l'échec des stratégies syndicales de type revendicatif est l'apparition d'une nouvelle confédération : le Dômei, issu en 1964 de la fusion entre le Zenrô-Kaigui et le Sôdômei ${ }^{25}$. La période des années 1960 marque donc la division du mouvement syndical, fondée sur un double clivage idéologique. 
Tout d'abord sur le plan politique, ce clivage repose sur la position vis-à-vis de la reconduction du traité de paix nippoaméricain. Il se traduit dès 1960 par la scission de l'aile droite du parti socialiste qui crée une nouvelle formation politique : le parti socialiste démocratique (PSD). La confédération syndicale du Dômei va soutenir la position du PSD, plus favorable à la politique gouvernementale. En fait, le Dômei critique la trop forte intervention du Sôyhô sur la scène politique. En revanche, le Sôhyô est solidaire du Parti socialiste japonais (PSJ). Ils prennent très tôt une position commune, avec l'adoption des quatre principes de paix réaffirmant la neutralité du Japon ${ }^{26}$.

De même, le Sôhyô a joué un rôle important pour l'organisation d'un mouvement d'opposition à la révision du traité de paix nippo-américain (1959-1960) ${ }^{27}$. Le Sôhyô demeure toujours un appui important du PSJ, tant du point de vue financier que du point de vue de la structure militante. Enfin, sur le plan de l'action syndicale, les points de vue divergent. Si le Sôhyô concentre son action sur le Shuntô, le Dômei adopte une position plus réservée. Il s'orientera vite au début des années 1970, vers une attitude de modération salariale.

Ainsi, le syndicalisme de coopération semble davantage adapté au nouveau type de relation salariale où le système de promotion est contrôlé par la direction. En acceptant cette situation de fait, le syndicalisme de coopération s'y est doublement associé. Tout d'abord, dans la mesure où ce type de relation salariale encourageait l'élévation" volontaire"28 par les salariés de la productivité, le syndicalisme de coopération ne s'est pas opposé à la politique de rationalisation. Il s'y est également associé d'un point de vue idéologique. Le syndicat de coopération insiste sur l'intérêt commun qui existe entre les salariés et la direction. La prospérité des salariés, c'est-à-dire la sauvegarde de l'emploi, est associée aux résultats de l'entreprise. 
En revanche, ce type de syndicat réclame une politique de concertation pour retrouver par son association aux décisions du personnel, une certaine audience vis-à-vis de sa base syndicale : les ouvriers qualifiés. Dans près de $90 \%$ des grandes entreprises, les commissions paritaires d'entreprises offrent un lieu de rencontre privilégié où les questions du personnel sont abordées ${ }^{29}$. Par une politique de concessions négociées, le syndicat de coopération entend aussi protéger le noyau dur du salariat.

La recomposition du paysage syndical se fait au profit des syndicats de coopération et voit l'érosion de l'influence du Sôhyô dans le secteur privé, où seules subsistent ses fédérations qui adoptent une politique de modération. Le seul examen des effectifs de chaque confédération nous confirme cette transformation. Ainsi le Dômei, qui avait débuté avec 1 million de membres voit ses effectifs doublés en 1970 (près de 2 millions). En revanche, moins de la moitié des membres du Sôhyô, dans les années 1970, appartient au secteur privé, tandis qu'il domine dans le secteur public. Quant au Churitsu Rôren, il regroupe près de 1,4 millions de membres.

Mais, dans le secteur privé, près de la moitié des syndicats d'entreprises ne sont affiliés à aucune confédération nationale. En fait, le Dômei ne représente que $21 \%$ environ des effectifs syndiqués du secteur privé, tandis que le Sôhyô n'en regroupe que $17 \%$ environ.

Comment peut-on expliquer une telle faiblesse des structures fédérales ? Elle tient en fait à la caractéristique de la forme syndicale au Japon : sa décentralisation. La structure de base demeure le syndicat d'entreprise, c'est à ce niveau que se regroupe dans une même organisation cadres, employés et ouvriers. Toutefois, si les fédérations (Tan-san) existent aussi au Japon, le lien entre celles-ci et les syndicats d'entreprises 
restent relativement souple. Il en est de même entre les fédérations et les quatre confédérations au niveau national.

Cette caractéristique de la forme syndicale au Japon reflète la nature de la relation salariale dans les grandes entreprises : un système de promotion intégré qui lie le salarié à l'espace d'une entreprise.

L'importance du syndicat d'entreprise est à la fois un élément de force et de faiblesse du syndicalisme japonais. Elément de force, car souvent les grandes entreprises pratiquent la clause de l'union-shop qui rend presque automatique l'affiliation au syndicat. Ainsi cet ancrage au niveau de l'entreprise a limité l'érosion des effectifs dans les secteurs traditionnels d'implantation syndicale.

Elément de faiblesse enfin, car le syndicat d'entreprise ne s'étend qu'au noyau dur du salariat : les employés permanents des grandes entreprises, en excluant les catégories de maind'oeuvre périphériques qui forment pourtant près de $70 \%$ de la population active ${ }^{29 b i s}$. Ainsi dans l'état actuel, les possibilités d'extension du syndicalisme se trouvent dépendre de la grande entreprise.

Par ailleurs, l'identité du syndicat d'entreprise, plus particulièrement du syndicalisme de coopération, se confond avec l'image de l'entreprise. De ce fait, l'identification du salarié permanent se fera davantage par rapport à l'entreprise qu'au syndicat. Dans le cas extrême, mais pourtant fréquent pour le syndicalisme de coopération, l'affiliation syndicale ne représente seuiement qu'un moyen de promotion rapide dans la hiérarchie de l'entreprise.

Au début des années 1970, le mouvement syndical au Japon offre une situation contrastée. D'une part, le syndicalisme de 
revendication est en crise, avec l'érosion des positions du Sôhyô dans le secteur privé. De l'autre, le syndicalisme de coopération semble prendre son essor, en s'appuyant sur la structure de base de l'entreprise.

Toutefois, cette mutation du mouvement syndical au Japon apporte aussi des éléments de faiblesse. Elle consacre, tout d'abord, la division du mouvement syndical et réduit ainsi son pouvoir de négociation déjà faible, au niveau national. De même, l'orientation des syndicats vers la coopération fait disparaître toute autonomie idéologique vis-à-vis de l'entreprise. La perte d'identité du syndicat, si elle peut être utile pour son développement au niveau local, n'est certainement pas favorable au renforcement de ces structures fédérales et confédérales. Enfin, l'exclusion de fait des catégories de maind'oeuvre autre que le noyau dur du salariat des grandes entreprises, aussi bien pour le syndicalisme de coopération que de revendication, est un élément latent de faiblesse ${ }^{30}$. L'arrivée de la crise en sera le révélateur.

1.3. Les pouvoirs publics : a la recherche de relations du travail "modernes"

Dans le cadre de la haute croissance, le marché du travail était en situation de pénurie de main-d'oeuvre. Les pouvoirs publics ont eu deux objectifs : accroître les ressources en maind'oeuvre pour détendre le marché du travail, améliorer le partage de la valeur ajoutée.

L'arrivée du premier ministre Ikeda, avec le lancement du plan de doublement du revenu, annonce aussi le thème du partage des fruits de la croissance. Cette philosophie, qui détermine le partage de la valeur ajoutée, selon les gains de productivité, trouvera un consensus du côté des employeurs comme des organisations syndicales, jusqu'au début des années 1970. En 1960, la loi sur le salaire minimum crée un salaire 
minimum local qui est fixé pour chaque aire géographique par le ministère du travail. Pour accroître l'offre de main-d'oeuvre, les pouvoirs publics mettent en place un dispositif législatif comme la loi sur la répartition de l'emploi (Kôyô-Taisaku-hô 1960) ou la loi sur la sécurité et la santé dans le travail (1972) pour assurer une main-d'oeuvre disponible toujours croissante.

Mais les principales transformations apparaissent avec la reconnaissance par les pouvoirs publics de la nécessité de relations du travail "modernes", c'est-à-dire fondées sur la négociation collective. En 1966, la commission du droit pour les relations industrielles réclame un réglement des conflits, non plus par la confrontation, mais par la négociation entre syndicats et employeurs.

En revanche, sa position pour le secteur public a été plus défensive, ne reconnaissant ni le droit de grève des fonctionnaires, ni leur droit à la négociation ${ }^{31}$. Ce sont les actions répétées des fédérations adhérentes au Sôhyô et l'évolution de la jurisprudence de la cour suprême (1966) qui vont permettre la reconnaissance des droits à la négociation collective dans le secteur public. Ainsi en 1964, le premier ministre déclare que la détermination des salaires dans le secteur public devra suivre les résultats du Shuntô du secteur privé.

Pour cette période, les relations du travail se fondent sur le réglement des conflits par la négociation collective, mais de type informel. Ainsi, au niveau national, les pouvoirs publics ont tenté de faciliter l'échange de points de vue en créant une table ronde tripartite, le Sankoron (Industry and Labor round table conference). Présidé par le Ministre du travail, elle réunit des représentants du gouvernement, des quatre confédérations syndicales, ainsi que les organisations patronales telles que le Nikkeiren, le Keidanren ou le Nissho (annexe : 1), pour aborder 
les principaux problèmes relatifs aux relations du travail. Toutefois, ces consultations ne débouchent pas sur un cadre contractuel. En fait, les conditions économiques de la haute croissance n'impliquaient pas une intervention directe de l'Etat qui se contentait d'offrir un cadre législatif fondé sur des recommandations plus que sur des obligations.

2. Les transformations de l'environnement économique et leurs effets sur l'audience des syndicats

2.1. La crise économique et l'abandon de la philosophie du partage des résultats

En 1974, l'offensive du printemps entraîne une augmentation sans précédent des salaires (près de $30 \%$ ), mais marque en même temps un tournant. La crise va émousser l'efficacité des négociations salariales et remettre en cause la philosophie du partage des résultats qui prévalait encore au début des années 1970. Aussi, les gains salariaux ne vont cesser de se réduire. La hausse des salaires n'est plus que de $9 \%$ entre 1975 et 1978 et tombe à $4 \%$ pour les années 1980. La perte d'efficacité du Shuntô se traduit par une baisse constante de la part du travail dans la valeur ajoutée dès 1974 (graphe 3). On constate également une réapparition des disparités selon les secteurs et la taille des entreprises (graphe 4).

Pour 1987, la hausse moyenne des salaires est de 3,5\% contre $4,5 \%$ en 1986. On distingue une disparité entre le secteur manufacturier et non manufacturier. Les hausses atteignent $2 \%$ pour les secteurs en crise tels que les chantiers navals ou la sidérurgie et $6 \%$ pour le secteur des services en expansion comme la communication ou les services financiers. Ainsi la norme globale de fixation des salaires qui était apparue au début des années 1960 tend à disparaître. 
Si la crise économique explique en grande partie la baisse d'efficacité du Shuntô, elle provoque également un ajustement des stratégies des partenaires sociaux.

Les organisations patronales remettent rapidement en cause la conception du partage des résultats pour y substituer une autre philosophie : la limitation des salaires selon la hausse de la productivité. Ce changement d'orientation s'effectue dès 1970 avec la parution d'un livre blanc sur la politique des revenus dans les pays étrangers. Les conclusions de cette étude insistent sur la primauté de l'intérêt général et la nécessité d'une consultation pour la fixation des salaires. Pour les employeurs, cette dernière doit être fonction de l'évolution de la productivité.

De même, pour répondre au Shuntô dans l'industrie sidérurgique, le patronat a adopté, très tôt, une tactique symétrique à celle des syndicats regroupant ses propositions de hausse au même moment. Les employeurs ont adopté la stratégie d'une offre d'augmentation salariale unique et définitive (tactique de la réplique en un seul coup). Ainsi, cette méthode de négociation rendait les grèves inutiles. Peu à peu, les syndicats de coopération comme les fédérations affiliées à l'IMF-JC ont accepté les augmentations salariales déterminées par les employeurs, sans déclencher de grèves.

En fait, les organisations patronales ont toujours mis l'accent sur deux éléments : d'une part, elles souhaitent que la négociation se déroule au niveau de l'entreprise pour tenir compte de ses capacités de paiement ainsi que des résultats de la branche.

De l'autre, une part croissante du patronat considère que la hausse des salaires devrait être davantage différenciée selon le mérite des individus. Cette orientation ne ferait que refléter 
l'introduction croissante de l'avancement des salaires selon le critère du mérite.

Cette position se renforce, après la réévaluation du yen en 1987. Le Nikkeiren souligne les dangers qu'une désindustrialisation fait courir à l'emploi dans le cadre d'une croissance des investissements japonais à l'étranger. Il insiste sur la préférence de l'emploi à une augmentation des salaires ${ }^{32}$.

En revanche, pour les organisations syndicales, le Sôhyô, initiateur du Shuntô remarque que "ces dernières années le processus de détermination des salaires est progressivement influencé par la conjoncture et les performances des entreprises individuelles. "Aussi réaffirme-t-il la nécessité de changer le cadre de référence des négociations pour que la logique macroéconomique prévale"33. Par ailleurs, le Sôhyô souligne une faiblesse du Shuntô : la tendance des fédérations à se reposer sur l'ensemble du mouvement. Cette confédération souhaite également regrouper sur une même période les négociations salariales du secteur privé et du secteur public afin de renforcer son pouvoir de négociation.

Au contraire, les syndicats comme le Dômei pratiquent la modération des salaires pour la sauvegarde de l'emploi. Ainsi, dans certains secteurs touchés par la crise, des concessions salariales sont faites par ces syndicats pour éviter une réduction du volume de l'emploi.

Quant aux pouvoirs publics, en 1974, ils ont analysé la forte hausse des prix comme une inflation par les coûts provenant d'une hausse des salaires trop élevée. Cependant, ils estiment qu'une politique des revenus est inopportune car ils considèrent que la fixation des salaires reposent sur les forces du marché et la négociation collective. Ces dernières années, après les conflits commerciaux et le rapport Maekawa qui prône une 
croissance fondée sur la relance intérieure, les pouvoirs publics semblent plus favorables à une progression des salaires.

En définitive, les résultats modestes du Shuntô indiquent les limites d'une action syndicale trop centrée sur la revendication salariale. Si la philosophie du partage des bénéfices pouvait parfaitement fonctionner en période de haute croissance, son application semble difficile dans une situation où la maind'oeuvre est excédentaire et la croissance limitée. Cet affaiblissement dans les négociations salariales entraîne un changement de philosophie de la majorité des syndicats du secteur privé qui, en liant le niveau des revendications aux résultats de l'entreprise, renforce la dépendance des syndicats à cette dernière.

\subsection{La différenciation du salariat et la désyndicalisation}

Comme dans la plupart des pays européens, la désyndicalisation affecte également le mouvement syndical japonais. Si l'on examine ce phénomène sur une longue période, on observe après la pointe exceptionnelle de l'après-guerre (en 1948 , le taux de syndicalisation était de $55,8 \%$ ), une stabilisation du taux de syndicalisation aux alentours de 35,5\% dans les années 1970. Le phénomène de décroissance débute avec la crise à partir de 1973 où le taux de syndicalisation chute légèrement à 33,1\%. Mais c'est surtout dans les années 1980 que l'on assiste à une dégradation lente et progressive du taux de syndicalisation qui atteint $27,6 \%$ en 1987 (tableau 1).

Toutefois, la décrue du nombre des adhérents est plus tardive et ne semble pas très significative. Ainsi les effectifs continuent à progresser faiblement jusqu'en 1975. Au début des années 1980, une baisse légère s'amorce, aggravée par une nouvelle décrue à partir de 1984 . 
Pourtant, au regard de ces évolutions, on ne peut pas parler d'une érosion mais davantage d'une stagnation ou d'une conservation des effectifs. En effet, si l'on examine seulement les années 1980, la perte du nombre d'adhérents représente $0,2 \%$ de l'ensemble des effectifs. Ainsi, contrairement à certains pays européens ${ }^{34}$, les syndicats au Japon ont réussi à préserver leurs effectifs.

Mais, si la crise est moins marquée elle n'en est pas moins réelle. La baisse constante du taux de syndicalisation indique le recul du fait syndical dans son ensemble. Pour tenter d'en dégager ces fondements, il nous faut donc examiner d'autres indicateurs.

On remarque tout d'abord que le taux de syndicalisation du secteur public (61,5\% en 1985) est relativement élevé par rapport aux taux de syndicalisation du secteur privé $(24,5 \%)$. Ainsi la politique de dérégulation du secteur public, bastion du syndicalisme de revendication, aura un impact non négligeable sur l'orientation du mouvement syndical.

Voyons ensuite le taux de syndicalisation par secteur : sa disparité est évidente. Il est particulièrement faible dans les services $(20,1 \%)$ et la distribution $(8,6 \%)$, tandis qu'il demeure relativement élevé pour le secteur manufacturier $(34,2 \%)$ (tableau 1). Si l'on examine son évolution, on observe une baisse importante pour les secteurs bancaires, ceux des transports et de la communication. En revanche pour le secteur manufacturier, le taux de syndicalisation décroît légèrement mais le nombre des adhérents progresse.

Face à cette évolution, on remarque en fait que la désyndicalisation reste faible dans le bastion traditionnel de l'implantation syndicale : le secteur manufacturier. Ceci est dû principalement à la très large diffusion de la clause de l'union- 
shop dans les grandes entreprises de ce secteur. Ainsi, le maintien des effectifs est en partie due à une raison institutionnelle.

En revanche, le faible taux de syndicalisation dans les nouveaux secteurs marque l'incapacité des syndicats à étendre leurs structures. Comment peut-on expliquer cette difficulté ? Si l'on examine le taux de syndicalisation par catégorie de maind'oeuvre, on remarque que ce dernier est très faible pour les femmes, les employés à temps partiel et les cols blancs (tableau 2).

Par ailleurs, le taux de syndicalisation pour les PME est relativement plus faible que celui des grandes entreprises. De plus il a tendance à se réduire, tandis qu'il augmente pour les entreprises de plus de 500 salariés (tableau 1).

Les nouveaux secteurs tels que les services, la distribution ou même les banques regroupent un certain type de maind'oeuvre. Ainsi, ce sont souvent des secteurs fortement féminisés, où la part des employés à temps partiel ainsi que celle des cadres est relativement forte, comparée aux secteurs traditionnels. La part des PME y est aussi prédominante.

Rappelons que la base des syndicats est constituée par le noyau dur des salariés permanents des grandes entreprises et que les autres catégories de main-d'oeuvre sont exclues. La main-d'oeuvre de ces nouveaux secteurs n'est pas la clientèle naturelle des syndicats.

En définitive, la modification de la structure des emplois provoque, par l'extension des nouveaux secteurs, une baisse du taux de syndicalisation. 
Ce phénomène renvoie à la tertiarisation de l'économie japonaise, c'est-à-dire à la montée des services. Cette transformation de la structure économique entraîne la diversification du salariat avec l'arrivée de nouvelles catégories de main-d'oeuvre ${ }^{34}$.

La tertiarisation de l'économie japonaise pose un défi au mouvement syndical : s'implanter dans les nouveaux secteurs. Aura-t-il la capacité de s'adresser à de nouvelles catégories de salariés qui n'étaient pas considérées comme stratégiques dans le mouvement syndical?

La tertiarisation n'est pas le seul élément contribuant au déclin du fait syndical. Les organisations syndicales qui recrutent surtout auprès du noyau dur du salariat doivent faire face à une modification profonde de la relation salariale. Le mode d'intégration du salarié à la grande entreprise change, bouleversant les stratégies syndicales.

3. Le défi de la flexibilité et les transformations du mouvement syndical (années 1980)

\subsection{Le défi de la flexibilité}

Dans les années 80 , le mouvement syndical doit faire face à deux profondes transformations. D'une part, les pouvoirs publics mènent une politique de privatisation du secteur public qui heurte de front le syndicalisme de revendication et plus particulièrement le Sôhyô. De l'autre, face à une croissance instable, les entreprises adoptent une politique de flexibilité de la relation salariale. Ces politiques ont d'importantes conséquences sur la gestion des salariés. Comment évolue la relation salariale dans ce contexte et quelle est l'attitude des organisations syndicales face à ces transformations ? Mais surtout quels sont les enjeux, à long terme, pour les syndicats? 
Nous tenterons de formuler, autour de ces questions, quelques hypothèses.

Vers une relation salariale flexible

L'innovation technique et l'apparition d'une croissance modérée sont les principaux facteurs qui contribuent à l'assouplissement de la relation salariale. Après le milieu des années 1970, l'innovation technique est centrée sur l'introduction de la micro-électronique dans les entreprises. Le niveau de diffusion devient assez élevé dès les années 1980, aussi bien pour les grandes entreprises que pour les PME. De même, après le premier choc pétrolier, on assiste à une stagnation de la production qui entraîne un ajustement des effectifs dans les secteurs en crise. Enfin, du côté de l'offre de travail, le vieillissement de la population entraîne un accroissement des coûts salariaux et la diminution des possibilités de promotion pour les classes d'âge jeunes dont le niveau de scolarisation s'élève.

Dans cet environnement, l'entreprise se heurte à de nombreux problèmes de gestion de la main-d'oeuvre auxquels les syndicats se trouvent également confrontés. On remarque, en effet, une double transformation qualitative : l'assouplissement de la relation salariale pour les employés permanents et l'utilisation croissante des formes particulières d'emploi pour les grandes entreprises. On examinera tout d'abord les changements de la relation salariale pour les employés permanents.

$\mathrm{Du}$ point de vue des entreprises, l'introduction des changements techniques soulève une interrogation : "la maximisation" de la qualification. Ainsi la rapidité des changements techniques, en modifiant la structure des emplois, entraîne parfois une obsolescence des qualifications acquises 
selon l'expérience dans l'entreprise ${ }^{36}$. Dès lors, l'expérience qui s'identifie dans le cas du Japon à l'ancienneté ne signifie plus forcément un accroissement de la qualification. Mais au contraire pour certaines catégories d'emploi, elle représente une dévalorisation.

Dans le contexte d'un rythme rapide d'innovation et d'une main-d'oeuvre plus âgée, comment l'entreprise peut-elle résoudre le problème de la formation des salariés pour l'acquisition d'un nouveau type de qualification ? Quelles sont ses conséquences sur la relation salariale ? On peut observer deux cas de figures.

Le premier cas est illustré par la poursuite d'une politique d'intégration de la qualification, en intégrant la formation des salariés $^{37}$. Mais contrairement aux années 1960, où la forme de la pyramide des âges ${ }^{38}$ offrait une main-d'oeuvre jeune, facilement adaptable aux transformations technologiques, pour les années 1980 se pose le problème du vieillissement de la population active (graphe 5). Dans les entreprises, ce phénomène soulève la question de l'adaptation de cette fraction de la main-d'oeuvre à l'innovation.

Comment évolue la gestion de la main-d'oeuvre et quels sont ses répercussions sur la relation salariale ? Devant une réduction des possibilités de promotion, une inégalité des salariés pour s'adapter à l'innovation, certaines transformations apparaissent. Ainsi, la progression des salaires ne suit plus seulement le critère de l'ancienneté, mais également le critère de la compétence individuelle. De même, les possibilités de promotion à des postes de responsabilité s'amenuisent ${ }^{39}$. On observe donc une dissociation des trois éléments que sont la progression des salaires selon l'ancienneté, la qualification et la promotion, qui étaient interdépendants et 
contribuaient à la notion de carrière. Cette dernière a perdu son unicité.

Le deuxième cas de figure est l'extériorisation des coûts de formation, par le recours au marché du travail pour faire appel à des spécialistes. - Ce cas semble fréquent pour les entreprises qui diversifient leurs activités $-^{40}$. Dès lors, ce type de gestion de la formation à des répercussions sur la relation salariale : il altère le principe de l'emploi à vie (ou plutôt du marché interne du travail) par le recrutement à mi-carrière, il extériorise le processus de la qualification. Dans ce cas, le contrôle de l'entreprise sur la qualification des salariés risque de disparaître et se pose à nouveau l'enjeu de la mobilisation des salariés. Cette pratique de location de main-d'oeuvre, qui était jusque-là illégale, est reconnue par les pouvoirs publics avec la loi sur les entreprises d'emploi temporaire du 5 juillet 1985.

Enfin, après 1974, la stagnation de la production oblige les employeurs à ajuster la masse de travail à la baisse d'activité. De nombreux moyens ont été utilisé. Toutefois deux d'entre eux ont entraîné une grande flexibilité de la relation salariale : les mesures de pré-retraites volontaires et la politique de transfert de main-d'oeuvre. La pratique des pré-retraites volontaires fut fortement utilisée après le choc pétrolier de 1975, altérant le principe de la sécurité de l'emploi jusqu'à la retraite ${ }^{41}$.

De même, la pratique des transferts de postes est devenu fréquente ${ }^{42}$. Ces changements de postes, ainsi que les transferts (Shukko) vers un autre établissement du groupe ou une entreprise sous-traitante, provoque une mobilité géographique qui conduit à une dégradation des conditions de vie. Cette pratique de prêt de main-d'oeuvre fonctionne comme un processus de diffusion des savoir-faire dans le groupe, mais permet également une réduction des licenciements. Du côté de l'entreprise, la politique des transferts de main-d'oeuvre 
implique que l'espace juridique de gestion n'est plus ni l'établissement ni l'entreprise mais s'étend de plus en plus au groupe ${ }^{43}$.

Ainsi, comme on l'a souligné précédemment, la rapidité des changements techniques et la stagnation de certains secteurs, entraînent un assouplissement de la relation salariale pour les employés réguliers. Assouplissement géographique d'une part avec la procédure des transferts, assouplissement de l'intégration à l'entreprise de l'autre, par l'extériorisation dans certains cas de la formation ou par la rupture de la notion traditionnelle de carrière. Ces observations ne s'appliquent uniquement qu'au marché interne du travail des grandes entreprises.

Il nous reste à examiner les changements intervenus dans l'utilisation de la main-d'oeuvre périphérique. On remarque tout d'abord une progression de nouvelles formes atypiques d'emploi telles que le travail à temps partiel, ou à durée déterminée. Ainsi la part des employés à temps partiel, dont la durée de travail est inférieure à 35 heures par semaine, passe de $6,3 \%$ en 1960 à $11,7 \%$ en 1986 . Cette tendance est en relation avec l'arrivée croissante des femmes sur le marché du travail, puisque près de $22,6 \%$ de ces dernières étaient en 1986, des employées à temps partiel.

Enfin, on assiste à un recours croissant à la sous-traitance, soit en extériorisant des activités, soit en introduisant, pour les tâches périphériques, une main-d'oeuvre en sous-traitance dans les grandes entreprises ${ }^{44}$.

Ces évolutions conduisent à deux observations : d'une part, les employés permanents représentent une catégorie de plus en plus marginale dans la population salariée. De l'autre, la relation salariale tend à se diversifier avec l'utilisation 
croissante des catégories de main-d'oeuvre. Ces éléments concourrent à l'érosion de la forme syndicale, centrée sur les employés permanents.

La politique de dérégulation des pouvoirs publics

La politique de dérégulation comporte deux volets : la privatisation des entreprises publiques (comme NTT, la régie des tabacs, les chemins de fer publics) et l'introduction des principes de gestion privée dans le secteur public. Cette orientation a un double impact : d'une part, la centrale du Sôhyô, jusque là fortement implantée dans le secteur public, est menacée par les privatisations. Ses tentatives pour résister se heurtent à l'opinion publique favorable à un allégement des dépenses de l'Etat. Les luttes des fédérations des chemins de fer publics et leurs échecs indiquent bien la marge de manoeuvre étroite dont dispose le syndicalisme de revendiation retranché dans le secteur public. De l'autre, la réduction du secteur public diminue les effets du Shuntô pour les négociations salariales du secteur privé. Mais c'est l'affaiblissement du syndicalisme de revendication dans le bastion du secteur public qui demeure le fait essentiel de ces dernières années.

\section{L'attitude des syndicats}

Quelles sont les conséquences des changements de gestion de la main-d'oeuvre sur les syndicats ? Dans quelles mesures acceptent-ils ces transformations?

Selon de nombreuses enquêtes qui ont été menées dans les années 1980 par l'Institut du Travail du Japon et la fédération de l'industrie électronique ${ }^{45}$, on constate que les syndicats de 
coopération sont, sous certaines conditions, en majorité favorables à l'introduction de nouvelles technologies ainsi qu'à la politique de transfert ${ }^{46}$.

Ces conditions se résument en quatre points : la conservation de l'emploi, la non-aggravation des conditions de travail et de salaire, la formation des personnels déplacés, la consultation préalable des syndicats. Axé sur une politique de négociation, le syndicat de coopération considère le plus souvent que ces mesures sont indispensables à la compétitivité de l'entreprise.

En revanche, les syndicats de revendication ont une attitude plus réservée vis-à-vis de l'introduction de la microélectronique. Ainsi seulement près de $32,4 \%$ y sont par principe favorable, tandis que près de $47,2 \%$ s'y résignent contre $8,3 \%$ qui y sont franchement opposés.

En ce qui concerne l'introduction de nouvelles technologies, il semble donc que se dessine le même type de clivage entre les syndicats de coopération et les syndicats de revendication, quoique plus nuancé.

En revanche, par rapport à la pratique des pré-retraites, les réticences syndicales sont plus importantes. Ceux-ci réclament, au contraire, l'allongement de l'âge de la retraite pour les employés permanents. Cette revendication qui semble paradoxale, permettrait en fait d'étendre le bénéfice de la sécurité de l'emploi à des classes d'âge où les chances d'un deuxième emploi s'amenuisent ${ }^{47}$.

De même, les syndicats se sont opposés, mais en vain, à la législation concernant les entreprises d'emplois intérimaires, qui altèrent le système de sécurité de l'emploi par la précarisation des postes. 
Au-delà de la seule attitude des syndicats vis-à-vis de l'introduction des changements techniques, on peut s'interroger concernant l'impact de l'assouplissement de la relation salariale sur les stratégies syndicales. La politique de transfert entraîne une gestion de la main-d'oeuvre au niveau du groupe et non plus de l'entreprise. Cette structure semble dépassée pour assurer une mobilisation efficace des salariés. De même, l'usage croissant d'une main-d'oeuvre périphérique, exclue du syndicat, réduit la mobilisation des salariés à la catégorie de plus en plus restreinte des salariés permanents.

Enfin, l'éclatement de la notion de carrière avec la dissociation de ses composantes, entraîne pour les salariés permanents une intégration d'un type différent. En fait, on assiste à un décalage entre la philosophie de l'entreprise qui reste inchangée et les transformations actuelles de la relation salariale. Quels seront les effets de cette ambiguité sur les syndicats de coopération majoritaire dans le secteur privé ? Va-t-on assister à une démobilisation croissante des employés permanents? Autant de questions qui restent en suspens.

D'ailleurs certains résultats d'enquêtes d'opinion semblent indiquer que le mode de participation aux activités syndicales s'éloigne de la forme militante et devient plus passive. Ainsi l'adhésion au syndicat est davantage considérée comme une attente de services, au même titre qu'une adhésion à une mutuelle ${ }^{48}$.

En tout cas, les organisations syndicales, conscientes de leurs faiblesses et des dangers que comporterait une plus forte démobilisation des salariés, cherchent des solutions. Les efforts pour unir le mouvement syndical semble être la voie choisie pour relever les défis de la flexibilité. 


\subsection{Les transformations du mouvement syndical}

\subsubsection{La naissance du Rengo}

Les syndicats, face à la désaffection des salariés qui menace leur influence sociale et politique, ont réagi en s'acheminant vers une union dans une confédération nationale commune.

Un environnement favorale à sa création

En effet, on constate l'épuisement, aussi bien des stratégies du Sôhyô orientées vers des gains salariaux, que des stratégies des syndicats de coopération tournées vers une politique défensive de concessions négociées. C'est pourtant dans ce contexte de crise des stratégies qu'apparaissent des facteurs favorables à l'union du mouvement syndical.

Sur la scène économique tout d'abord, afin de réduire les conflits commerciaux, la politique économique s'oriente vers l'encouragement de la demande intérieure (rapport Maekawa). Or, cette réorientation semble favoriser, au niveau des priorités gouvernementales, la réduction effective de la durée du travail.

Ensuite certaines évolutions renforcent la nécessité d'une union du mouvement syndical. Ainsi le phénomène de délocalisation des activités économiques, accéléré après la réévaluation du yen, fait peser la crainte de l'accroissement du chômage, comme l'aggravation de la politique de tranfert de la main-d'oeuvre. Les organisations syndicales désunies s'inquiètent de leur incapacité à contrôler ce phénomène qui dépasse leurs structures trop décentralisées.

D'un autre point de vue, le vieillissement de la population active pose non seulement des problèmes au niveau de l'entreprise sur le plan de la formation, mais également au niveau national, sur le plan de la protection sociale. Enfin, le 
problème de la répartition des revenus, c'est-à-dire de la fixation des salaires, mais également de la fiscalité, est actuellement remis en cause par les pouvoirs publics.

Sur la scène politique on assiste de manière ponctuelle, à un certain rapprochement des partis d'opposition ${ }^{49}$ allant jusqu'à des actions communes contre le projet de réforme fiscale présenté par l'ancien premier ministre Nakasone.

Le changement de programme du PSJ vers un programme plus réformiste favorise sans doute ce rapprochement. Dans un contexte de cristallisation des demandes sociales, cet environnement semble plus propice à l'exercice d'une pression syndicale sur les partis politiques. Quant au PLD, certaines de ses fractions sont à la recherche d'une nouvelle base sociale pour remplacer des catégories menacées telles que les agriculteurs $^{50}$.

Sur le front syndical enfin, c'est l'affaiblissement continu du Sôhyô qui a abouti à un changement des rapports de force à l'intérieur du mouvement syndical. Ainsi, la politique de dérégulation menée par le gouvernement a affaibli le bastion du Sôhyô : le secteur public. Face aux risques de marginalisation, les instances dirigeantes du Sôhyô semblent être disposées à favoriser l'union du mouvement syndical.

Les étapes vers une union syndicale

La confédération des syndicats du secteur privé japonais (Rengo) s'est formée le 20 novembre 1987 réunissant 60 fédérations d'industries qui comptent près de 4,6 millions de travailleurs. Elle n'est que l'aboutissement d'un long processus de tentatives nombreuses menées tout d'abord à l'initiative des syndicats de coopération, dominant le secteur privé ${ }^{51}$. 
Une des premières étapes est marquée par la création du conseil national d'orientation des politiques (1976) puis du Zenmin Rôkyo (conseil japonais des syndicats du secteur privé), créé en 1983. Ces organisations regroupaient les principales fédérations du secteur privé dans le but de faire des propositions sur l'orientation générale de la politique gouvernementale. Toutefois le Zenmin Rôkyo n'était qu'une structure de concertation souple où les fédérations conservaient leur autonomie.

La création du Rengo marque une nouvelle étape vers une intégration des anciennes confédérations dans une structure commune. Pour l'instant, celles du Dômei, du Churitsu Rôren (1987) et du Shinsanbetsu (1988) se sont dissoutes pour s'intégrer au Rengo qui représente la plus large confédération existante au Japon dépassant le Sôhyô qui ne regroupe que 4 millions de membres.

Parmi les fédérations d'industries adhérentes au Rengo, toutes n'ont pas la même importance. Seules cinq parmi elles ont plus de 300.000 syndiqués $^{52}$. Trois de ces fédérations, syndicats de coopération puissants du secteur privé, ont eu d'ailleurs un rôle décisif dans la création du Rengo. Elles représentent en outre les secteurs traditionnellement exportateurs de l'économie japonaise.

Trois autres importantes fédérations, le Dentsu Rôren, le Zennitsu et Tabacco, représentent les entreprises publiques récemment privatisées. En fait, près de $74 \%$ des syndicats qui adhèrent au Rengo ont moins de 100.000 membres. Leur faiblesse numérique provient de la rivalité entre les deux centrales syndicales du Sôhyô et du Dômei. Cette rivalité entraîne des divisions et la non-adhésion des syndicats d'entreprises, ceux-ci s'opposant à l'orientation de leurs fédérations d'industrie. 
Cette division à la base réduit la taille des fédérations en affaiblissant leur représentativité et leur pouvoir de négociation. En dépit de son annonce, la création officielle du Rengo n'est encore que partielle. Si une étape décisive a été franchie, de nombreux obstacles demeurent encore pour une réelle unité du mouvement syndical.

Les objectifs et obstacles du Rengo

Les difficultés que rencontre la réalisation de cette union proviennent essentiellement du clivage idéologique qui traverse le mouvement syndical au Japon. Aussi après avoir examiné les objectifs énoncés par le Rengo, c'est à travers l'analyse des obstacles qui subsistent que l'on découvrira la lutte d'influence que se livrent toujours syndicalisme de coopération et syndicalisme de revendication.

L'objectif principal du Rengo est la création "d'un front uni du travail" pour dépasser les clivages du secteur privé et étendre cette réunion jusqu'au secteur public. En augmentant son pouvoir de négociation, le Rengo voudrait influencer la politique gouvernementale grâce au renforcement des liens noués avec les partis politiques.

Il permettrait d'offrir également un centre de réflexion et de recherche pour les fédérations adhérentes ${ }^{53}$.

Toutefois, pour réaliser ces objectifs le Rengo devra surmonter trois types d'obstacles : le problème de l'adhésion du Sôhyô, la question des relations avec les partis politiques et la réalisation d'une structure unique au niveau régional.

Si la dissolution du Sôhyô et son intégration au Rengo est en principe acquise, puisque celle-ci est programmée pour 1989 par ses dirigeants, elle ne va pas sans poser de problèmes. En 
effet, la question de l'intégration au Rengo divise le Sôhyô en deux grands courants qui s'affrontent. Les partisans de l'intégration se situent dans les principales fédérations du secteur privé : sidérurgie, chemins de fer privé et télécommunication ${ }^{54}$. Ces dernières représentent la presque totalité des effectifs du Sôhyô dans le secteur privé et constituent, pour celui-ci, un soutien financier non négligeable.

Par ailleurs, la politique de privatisation des pouvoirs publics pousse certaines fédérations du secteur public à rechercher une intégration au Rengo.

Ainsi, le Jichirô (syndicat des travailleurs municipaux et des préfectures du Japon), fédération influente du Sôhyô, est partisan de l'adhésion au Rengo. Face à la réforme administrative lancée par l'ancien premier ministre Nakasone, cette fédération, par son isolement, redoute son faible pouvoir de négociation.

Aussi, son adhésion au Rengo semble être une alternative qui renforcera sa position grâce au soutien présumé des fédérations du secteur privé majoritaires dans le Rengo. Cette stratégie tente d'éviter l'affaiblissement du secteur public due à l'orientation de la réforme administrative qui cherche à opposer les résultats de sa gestion à ceux du sècteur privé.

Les opposants à l'intégration au Rengo se forment de deux courants. L'un est composé de syndicats influencés par le Parti communiste japonais (PCJ), qui représente au sein du Sôhyô, le courant anti-majoritaire. L'autre groupe est formé du courant majoritaire de gauche affilié au Parti socialiste. Ces deux groupes s'opposent à l'orientation modérée prise par les dirigeants du Sôhyô, influencés par les grandes fédérations du secteur privé. 
En général, ces opposants ne sont pas regroupés au sein de fédérations spécifiques, mais sont au contraire éparpillés, en courants le plus souvent minoritaires, dans plusieurs types de syndicats. Cette dispersion entraîne parfois une lutte d'inflence pour la prise de contrôle de certaines fédérations. En ce qui concerne le secteur public, ces conflits, qui sont le signe de la crise de l'institution syndicale face à l'offensive méthodique menée par les pouvoirs publics, renforcent l'affaiblissement de la fédération concernée ${ }^{55}$.

En effet, si le Rengo tente de dépasser les clivages idéologiques qui existent principalement entre le Dômei et le Sôhyô, c'est en excluant de ce front uni du travail la composante influencée par les communistes. Ce rejet apparaît d'une manière implicite dans une déclaration en 1986 qui sert de profession de foi pour le Rengo. L'article quatre précise que le Rengo se gardera de toute ingérence extérieure.

Pour les fondateurs du Rengo, l'exclusion des communistes permettrait de prévenir les risques de scission comme ceux qui ont marqué l'histoire du mouvement syndical au Japon. D'ailleurs, le PCJ a réagi en condamnant le réalignement du mouvement syndical à droite. De même, la création de la conférence des syndicats pour la promotion d'un front uni (Toitsu Rôso-Kon) a permis de regrouper, au-delà du Sôhyô, tous les syndicats d'obédience communiste.

Enfin les opposants rejettent les priorités d'action fixées par le Rengo qui privilégie davantage les renvendications sur la réforme fiscale, tout en considérant que l'action du Shunto pour la croissance des salaires se heurte aux exigences économiques et ne devient que secondaire. Les opposants au sein du Sôhyô estiment que cette orientation se réduit à passer un compromis avec les pouvoirs publics, pour accepter dans ses grandes lignes la réforme fiscale. 
Le Rengo est considéré par ses opposants comme une tentative pour étouffer un syndicalisme ancré à gauche et réduire le bastion du secteur public détenu par les fédérations les plus revendicatives du Sôhyô. Ainsi, cette confrontation qui divise le Sôhyô est une bataille idéologique qui remet en cause non seulement l'orientation de l'action syndicale mais également les relations avec les partis politiques.

L'attitude du Rengo vis-à-vis des partis politiques constitue également une pierre d'achoppement pour l'unité du mouvement syndical. Traditionnellement, chaque confédération importante avait des liens privilégiés avec un parti politique, apportant un soutien financier et recommandant ses candidats qui, une fois élus, les représentaient au sein du parti politique (tableau 3).

Cependant avec l'affaiblissement de l'audience des syndicats, ces partis politiques ont tenté de se dégager d'une influence trop forte des organisations syndicales pour élargir leurs bases électorales vers d'autres couches de la population ${ }^{57}$. Ainsi cet affaiblissement du rôle politique des syndicats se traduit par une baisse constante du nombre des députés élus à la diète nationale sur recommandation des syndicats ${ }^{58}$.

Par l'unification du mouvement syndical, le Rengo entend retrouver son pouvoir de négociation avec le gouvernement comme avec les partis politiques. Trois cas de figure se présentent : l'un est de tenter l'union des partis politiques d'opposition que sont le parti socialiste, le parti démocrate socialiste et le Komeito pour proposer une alternative à l'hégémonie du Parti démocrate libéral (LDP) au pouvoir.

L'opposition à la réforme fiscale lancée par Nakasone a été un banc d'essai pour tenter un premier rapprochement. Toutefois, celui-ci reste fragile et dépend de la force de 
proposition du PLD, qui par le jeu des compromis avec certains partis de l'opposition, cherche à obtenir un consensus.

Le premier ministre Takeshita a utilisé cette stratégie pour faire adopter le nouveau projet de réforme fiscale ${ }^{59}$. Par ailleurs, de nombreuses différences idéologiques, telles que le réarmement ou l'énergie nucléaire, demeurent un obstacle à l'union de l'opposition ${ }^{60}$.

Le deuxième cas de figure passe par une action sur certaines factions du PLD à la recherche d'un nouveau électorat urbain.

Enfin la dernière solution serait de prôner une indépendance d'action vis-à-vis des partis politiques. Dans ce cas l'influence sur l'orientation de la politique gouvernementale ne passerait plus par un soutien direct des partis politiques mais par un renforcement de l'action revendicative sur le terrain. Cette dernière suppose un regain de militantisme, un élargissement de l'audience et une transformation des structures syndicales qui paraissent problématiques.

En effet, cette stratégie pose le problème de l'union de l'action syndicale au niveau régional. Les structures du RENGO demeurent pour l'instant au niveau national. L'extension au niveau régional, jusqu'aux syndicats d'entreprises, suppose la disparition des conflits entre les syndicats affiliés aux instances régionales du Sôhyô (Chihô hyôgikai) et ceux affiliés au Dômei.

Cette extension suppose une union des actions revendicatives rendue très difficile à réaliser par la divergence des points de vue plus forte sur le terrain.

Ces obstacles à l'achèvement du Rengo révèlent la lutte d'influence qui se joue entre le syndicalisme de coopération et le syndicalisme de revendication. Si le syndicalisme de 
coopération a très tôt triomphé dans le secteur privé dès le milieu des années 1960, le secteur public demeure encore le bastion d'un syndicalisme de revendication. D'une part, dans le contexte d'une politique de dérégulation rapide, le syndicalisme du secteur public (c'est-à-dire principalement le Sôhyô) semble ébranlé. De l'autre, la création du Rengo, à l'initiative du syndicalisme de coopération du secteur privé ressemble pour certains à une tentative d'annexion du secteur public. Toutefois, l'issue de cette lutte d'influence est encore incertaine et l'importance de son enjeu donne déjà lieu à des tensions internes croissantes.

\section{POUR CONCLURE}

L'affaiblissement du mouvement syndical au Japon se manifeste par le phénomène de désyndicalisation relative et la baisse de son rôle social et politique. Il est la traduction d'une crise qui recouvre plusieurs aspects.

C'est tout d'abord la crise du syndicalisme de revendication qui a débuté très tôt pour le secteur privé, pour s'étendre ensuite au secteur public, avec la politique de dérégulation. Ce type de syndicalisme s'est orienté vers la recherche de gains salariaux, abandonnant la stratégie de contrôle en matière d'organisation du travail. L'éloignement des stratégies syndicales de l'entreprise et les politiques patronales d'éviction ont contribué à cette crise.

C'est ensuite la division du mouvement syndical, avec l'apparition d'un clivage idéologique entre syndicalisme de revendication et syndicalisme de coopération. Cette division a affaibli le pouvoir de négociation du mouvement syndical sur la scène politique. 
C'est surtout la crise de la forme syndicale. En effet, la différenciation du salariat, l'évolution des pratiques de gestion du personnel des grandes entreprises ont modifié la relation salariale. Une double transformation s'est produite : d'une part, une diversification des modes d'intégration à l'entreprise selon la catégorie de main-d'oeuvre, de l'autre, un assouplissement de la relation salariale dans les grandes entreprises.

Aussi, le syndicalisme d'entreprise qui correspondait à une relation salariale fondée sur un principe unique de promotion, se trouve ébranlé. Dans le cadre d'une plus grande flexibilité de la relation salariale, le maintien de l'audience syndicale dépend en grande partie de sa capacité à capter les différentes couches du salariat. Ceci ne va pas sans une transformation de la structure syndicale. Il est également fonction de la recherche d'une nouvelle identité, plus autonome par rapport à l'entreprise.

Aujourd'hui, les stratégies des syndicats se tournent davantage vers la scène politique. L'union du mouvement syndical, en dépit de ses difficultés, est une tentative pour accroître son pouvoir de négociation au niveau national. Parviendra-t-elle à éviter l'érosion de l'audience syndicale auprès des salariés, en dépassant les limites du syndicalisme d'entreprise ? Les résultats du Rengo dépendent aussi de son orientation et de l'alternative entre stratégie de revendication ou politique de coopération. Ces transformations ne manqueront pas d'influencer le jeu des partis politiques. 


\section{NOTES}

1. Cf. P. ROSANVALON, La question syndicale, Paris, Calmann- Levy, 1988, p. 23.

2. La relation salariale est définie ici comme le rapport qui lie le salarié à l'entreprise. Il exprime a la fois un mode de répartition, mais aussi une logique d'intégration/socialisation du salarié à l'entreprise. Cf. la présentation de J.J. SILVESTRE, "Le salaire chantier par une approche pluridisciplinaire", texte préparatoire aux Journées des économistes et sociologues du travail , Paris, novembre 1988.

3. Nippon Steel 1953.

4. Mitsui-Miike 1960.

5. Cf. K. KOIKE "Human Resource Development and Labor Management Relations" Discussion paper $\mathrm{n}^{\circ} 221$, Kyoto University 1987, p. 56.

6. Ce que certains appellent emploi à vie, d'autres le relient à l'analyse des marchés internes du travail : cf. H. SHIMADA "The Japanese Employment System", The Japan Institute of Labor serie : 6, 1980, p. 13.

7. H. KUMON.

8. La ligne de partage entre activité syndicale et activité d'encadrement, qui reste floue au Japon, est un enjeu dans la mobilisation des salariés pour les grandes entreprises.

9. Yawata Steel, 1958.

10. Par exemple chez Nippon Steel.

11. Ces groupes, appelés groupes d'autogestion (Kushu-Kanri) ont démarré dans les grandes entreprises de la sidérurgie dès les années 1960. Le mouvement des cercles de qualité succède à ces activités. 
12. I. SAGA "Qualifications mutations technologiques et pratiques syndicales", CRMSI, 1983, p. 13.

13. H. HIRATA, K. SUGITA, "Politique paternaliste et division sexuelle du travail : le cas de l'industrie japonaise", Le Mouvement social, $\mathrm{n}^{\circ}$ 144, juillet-septembre 1988.

14. Les prestations sociales représentent environ $13 \%$ du coat total du travail.

15. Tels que des prêts à taux préférentiel pour l'achat d'un logement, un systeme de soins, des maisons de vacances et cercles culturels.

16. Cette classification ne représente qu'un idéal-type des syndicats, pour saisir leurs grandes évolutions. Parmi les nombreuses typologies syndicales qui ont été élaborées, on choisira deux critères : l'un est l'idéologie conçue ici comme la représentation que le syndicat peut avoir de ses relations avec l'adversaire/protagoniste : soit des relations d'antagonismes, soit des relations de coexistence. Ces idéologies mettent en jeux les objectifs profonds des syndicats : changement du système social ou simple contrôle de ce dernier. Le deuxième critère est le type d'action syndicale : négociation ou grève par exemple. Cf. G. CAIRE, Les syndicats ouvriers, Paris, PUF, p. 277.

17. Article 3 de la charte du Sôhyô : "The interests of the working class are fundamentally opposed to those of the capitalist class"... Si la philosophie d'ensemble du Sôhyô est proche du syndicalisme de revendication, la faible centralisation des syndicats entraîne au niveau des fédérations une plus grande diversité. Ainsi le TekkoRoren, fédération affiliée au Sôhyô est plus proche du syndicalisme de coopération.

18. Article 4 de la charte du Dômei : "We will promote the modernization of industry in order to bring about continuous prosperity of our national economy and endeavor to establish a thoroughgoing industrial democracy by working out effective plans for raising productivity and actively participating in the implementation of those plans". F. AYUSAWA, A History of Labor in Modern Japan, Honolulu, Greenwood Press, 1966. 
19. Voir p. 1-2.

20. Voir pour le conflit de 1953 chez Nissan (Datsun), la description donnée par I. MUTTO "Lutte de classe et innovation technologique au Japon depuis 1945", Cahiers d'études et de recherches, $n^{\circ} 5,1987$, p. 19 : Les stratégies de réduction du monopole syndical par la direction de Nissan ont entrainé la disparition de la fédération nationale des travailleurs de l'automobile. Celle-ci refusait la politique de rationalisation conduisant à l'augmentation de la productivité et à la réduction à terme des effectifs.

21. Créé a l'initiative du Zensendomei, fédération de tous les travailleurs du textile, il regroupe le Zenei En, acteurs et le Zen nippon kaiin kumiai marins pêcheurs. Cette centrale est dirigée par Takita Minoru, ancien dirigeant de la fédération du textile.

22. H. TSUTOMU, "Participatory Management and Japanese Workers" in J. BERGMANN, T. TOKINAGA, Economic and Social Aspects of Industrial Relations, New York, Capus verleig, 1987, p. 261-270.

23. La chute de Minoru Takano remplacé par Otha et Iwai en 1955.

24. Pour une présentation du mouvement syndical dans les chemins de fer nationaux au Japon, voir M. HANADA, "Privatisation des chemins de fer et crise du syndicalisme cheminot au Japon", in Travail, n 14 , octobre 1987.

25. C'est-à-dire la droite du Sôdômei qui n'avait pas rejoint le Sôhyô en 1950.

26. La déclaration des quatre principes de paix, adoptée en 1951 :

1. la conclusion d'un traité de paix multilatéral avec également l'URSS,

2. une neutralité absolue,

3. pas de base militaires au Japon,

4. pas de réarmement (Ayusawa, p. 318, op.cit ., p. 5).

27. En dépit de la démission du premier ministre Kishi, la ratification du traité marque un arrêt du mouvement pacifiste au Japon. 
28. Ce type de relation salariale pousse les employés permanents à améliorer la productivité pour favoriser leur mobilité dans l'entreprise. Cf. K. KOIKE, "Human Resource Development and Labor Management Relations" in K. YAMAMURA and YASUBA, The Political Economy of Japan, Standford California, Standford University Press, 1987.

29. A. ISHIKAWA, "Le syndicalisme japonais dans un environnement en mutation" in Revue Internationale des Sciences Sociales, vol.: $36, n^{\circ} 2,1984$.

29bis. Le Sôhyô, Dômei, Shinsanbetsu et Churitsu-Rôren, cf. Annexe 1.

30. Les salariés des PME, les employés à temps partiel ou à durée déterminée.

31. La constitution du Japon garantit aux travailleurs "le droit de se syndiquer ainsi que de mener des négociations et des actions collectives". Toutefois, après l'annonce d'une grève générale en 1949, Mac Arthur supprime en 1950 le droit de grève et l'action collective pour les fonctionnaires.

32. Libre blanc sur les relations du travail, (Rôdô undô hakusho), Ministère du travail, Tokyo, 1988, p. 46.

33. Sôhyô News, n 392, p. 13.

34. Comme la France par exemple, cf. P. ROSANVALON, p. 14, op.cit., p. 1.

35. K. KOSHIRO, "Les relations du travail et les transformations de la structure induustrielle" (Sangyô Kôzô no Henka to Rôshi Kankei), Revue de l'Institut Japonais du Travail (Nihon Rôdó Kyôkai Zasshi), 1988, juin $\mathrm{n}^{\circ} 346$. 
36. On ne considérera pas ici le débat concernant les effets de l'innovation technique sur la structure des qualifications : l'alternative entre déqualification ou nouveau type de qualification. Il s'agit ici de faire un constat à court terme, où la rapidité des changements entraîne une dévalorisation immédiate de certaines qualifications traditionnelles.

37. Cette intégration de la formation est parfois très avancée dans certaines entreprises, où un système d'éducation selon la fonction et le rang est mis en place, par exemple dans les banques.

38. H. SHIMADA, p. 13, op.cit ., p. 2.

39. En 1978, près de $70 \%$ des employés ayant un grade universitaire obtenait un poste de responsabilité; en 1988 ils ne sont plus que $30 \%$.

40. Ce dernier cas de figure est très récent au Japon, où certaines entreprises pour faire face a leurs besoins de spécialistes dans les domaines tels que l'informatique, ont recours a des entreprises intérimaires. Une organisation patronale, le Keizai Doyu Kai a publié en 1984, un rapport sur "la promotion de la microélectronique et les relations industrielles", concernant le marché intermédiaire du travail (à l'intermédiaire entre le marché interne de l'entreprise et le marché externe). Celui-ci devrait résoudre, du point de vue des organisations patronales, les problèmes de l'introduction de la micro-électronique.

41. M. TSUDA, "Will life time employment security practives be kept in 1980's ?", in Hitotsubashi Journal of Social Studies, vol. 12.

42. Pour l'industrie électronique, près de $57 \%$ des établissements ont effectué des transferts de main-d'oeuvre dans la période de 1978 à 1982.

43. Aussi, pour éviter des problèmes juridiques, ces pratiques sont souvent prévues dans le contrat d'embauche. Pour Nippon Steel, par exemple, l'article $5 \mathrm{du}$ contrat de travail prévoit que selon les besoins de l'activité, les membres du syndicat d'entreprise pourront être appelés à travailler à l'extérieur de l'entreprise.

44. Ainsi, le taux de sous-traitance était de $53,3 \%$ en 1976 pour 
l'ensemble des secteurs manufacturiers, il représente près de $65,5 \%$ en 1981. Dans certains secteurs, comme la sidérurgie, la soustraitance de main-d'oeuvre (Shagairo) représente près de $40 \%$ des effectifs.

45. L'Institut du travail a mené deux enquêtes, l'une en 1980, l'autre en 1984, concernant les restrictions que les syndicats émettaient pour l'introduction de la micro-électronique.

46. Pour l'enquête de 1984 , près de $53,6 \%$ de l'ensemble des syndicats d'entreprises interrogés sont fondamentalement favorables à l'introduction de la microélectronique, tandis que $36,6 \%$ pensent qu'elle est inévitable. Seuls $2,2 \%$ y sont fondamentalement opposés. Les syndicats de coopération, tels que le Dômei, y sont favorables a près de $67,5 \%$ l'adoption de la micro-électronique et les politiques qu'ils proposaient pour s'adapter à leur introdution, in Microelectronics and the Response of Labor Unions, The lapanese Institute of Labour, p. 1.

47. Le Ministère du travail a d'ailleurs reculé l'âge de départ obligatoire de la retraite qui se situait entre 55 ans et 60 ans et attribué une aide financière pour les entreprises qui conservaient cette catégorie de main-d'oeuvre.

48. Enquête du Sôhyô sur l'image des syndicats (septembre 1987), enquête du Rengo sur l'image des syndicats pour les jeunes (novembre 1988), pour un échantillon de 396.939 personnes et un taux de réponse de $75 \%$.

49. Komeito, Minshato (PSD), Shakaito (PSJ), cf. Annexe 1.

50. Les agriculteurs, base sociale traditionnelle du PLD, sont en effet menacés par l'ouverture des marchés agricoles.

51. Pour le secteur privé on retrouve : la fédération japonaise des syndicats de l'outillage électrique (Denkirôren), la confédération japonaise des syndicats de l'automobile (Jidôsha Rôren), ainsi que la fédération nationale des travailleurs du textile (Zensen Dômei). 
52. M. NITTA, "Birth of Rengo and Reformation of Union Organization", February 1, 1988, Japan Labor Bulletin .

53. Le Tekko-Rôren (fédération japonaise des travailleurs de la sidérurgie), le Shitetsu-Rôren (fédération japonaise des syndicats des travailleurs des compagnies de chemins de fer privés) et le Zendentsu (syndicat japonais des travailleurs des télécommunications), celles-ci ont d'ailleurs adhéré au Rengo tout en maintenant leur appartenance au Sôhyô. Cette double adhésion ne va pas sans soulever des problemes financiers qui poseront a terme le choix d'une confédération unique.

54. "Quels sont les objectifs du Rengo" (Rengo nani wo mesasu ka) Revue trimestrielle du droit du travail , (Kikan Rôdô Hô), $n^{\circ} 146$, 1988 , p. 125.

55. Le PSJ pour le Sôhyô, tandis que le Dômei soutenait le PSD.

56. Ce scénario d'affaiblissements successifs s'est déjà déroulé plusieurs fois. On citera le cas des chemins de fer nationaux avant leur privatisation. C'était également au sein du Syndicat des enseignants japonais (Nikkioso) que se déroulait une confrontation entre deux tendances : l'une modérée, l'autre de gauche qui exige la démission de son dirigeant, favorable au Rengo. Il semble que ce dernier courant s'est imposé. Ce conflit était l'illustration des difficultés que le Sôhyô rencontre pour définir un consensus autour de l'intégration au Rengo.

57. Ainsi le parti socialiste tente de se libérer de l'emprise du Sôhyo pour étendre son audience à d'autres groupes tels que les femmes, ou les mouvements de citoyens. L'élection comme secrétaire général d'une femme, Doi Takako, marque ce tournant.

58. A. ISHIKAWA, op.cit ., p. 9.

59. Ainsi, pour faire passer le projet de loi sur la réforme fiscale, le PLD accepte la demande des partis d'opposition et donne la priorité au projet de loi sur les réductions fiscales, in Japan Economic Journal, July 23, 1988. 
60. Par exemple, concernant le réarmement du Japon. Ce problème provoque d'ailleurs une opposition entre l'aile droite et l'aile gauche du Parti socialiste. Libre Blanc des Relations du Travail , p. 4, op.cit ., p. 13.

\footnotetext{
ANNEXE

Confédérations syndicales :

DOMEI : la confédération japonaise du travail (1964), président : T. OUSAMI.

SOHYO : (Nihon Rodo Kumiai Sôhyô Guikai). Le conseil général des syndicats japonais (1950), président : T. KUROKAWA.

CHURISTU-ROREN : la fédération des syndicats indépendants, président : $M$. WARASHINA.

SHINSANBETSU : (Zen Nihon Kinzoku Sangyô Rôdô Kumiai Kyoguikai). La fédération nationale des syndicats indépendants (1945), président : A. KAWAI.

Nouvelles centrales :

KINZOKU ROKYO (IMF-JC) : le comité japonais des fédérations métallurgistes internationales affilié à la CISL.

ZEMMIN ROKYO : le conseil des syndicats japonais du secteur privé (1983).

RENGO : (Zen Nihon Minkan Rodo Kumiai Rengo Kai). La confédération japonaise du secteur privé (1987), président : $T$; TAKASHIYAMA.
} 
Organisations patronales :

KEIZAI DOYUKAI : le comité japonais pour le développement économique, président : J. ISHIHARA.

NIKKEIREN : (Nihon Keieisha Dantai Rengo Kai). La confédération des associations patronales japonaises.

NIHON SEISAN HONBU : le centre japonais de la productivité.

NISSHO : Chambre de commerce et d'industrie japonaise, président : $R$. ISHIKAWA.

KEIDANREN (Keizai Dantai Rengo Kai), fédération des organisations économiques du Japon, président I. SAITO.

\section{PARTIS POLITIQUES}

PARTI LIBERAL DEMOCRATE (JIMINTO) : issu de la fusion du parti libéral et du parti démocrate en 1955.

PARTI SOCIALISTE JAPONAIS (SHAKAITO) : fondé en novembre 1945. En 1987, Takako Doi, première femme à diriger un grand parti au Japon remplace $M$. Ishibashi destitué en raison de la défaite électorale.

PARTI COMMUNISTE (KYOSANTO) : fondé en 1922, légalisé en 1945, son président est K. MIYAMOTO.

PARTI KOMEITO (parti du gouvernement propre) fondé en 1964, issu de la secte boudhiste Sokagakkai. 
Graphe 1 : Conflits du travail (1951-1970)

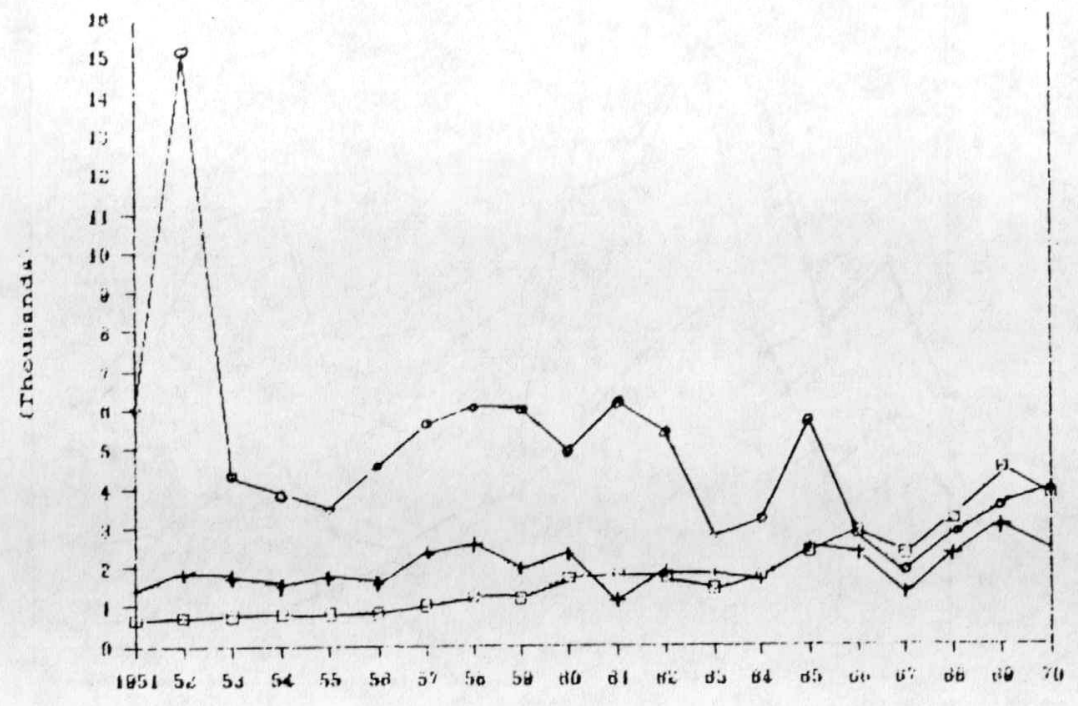

Conflits + Nbre de salariés Nbre de jours 
Graphe 1bis : Conflits du travail (1970-1984)

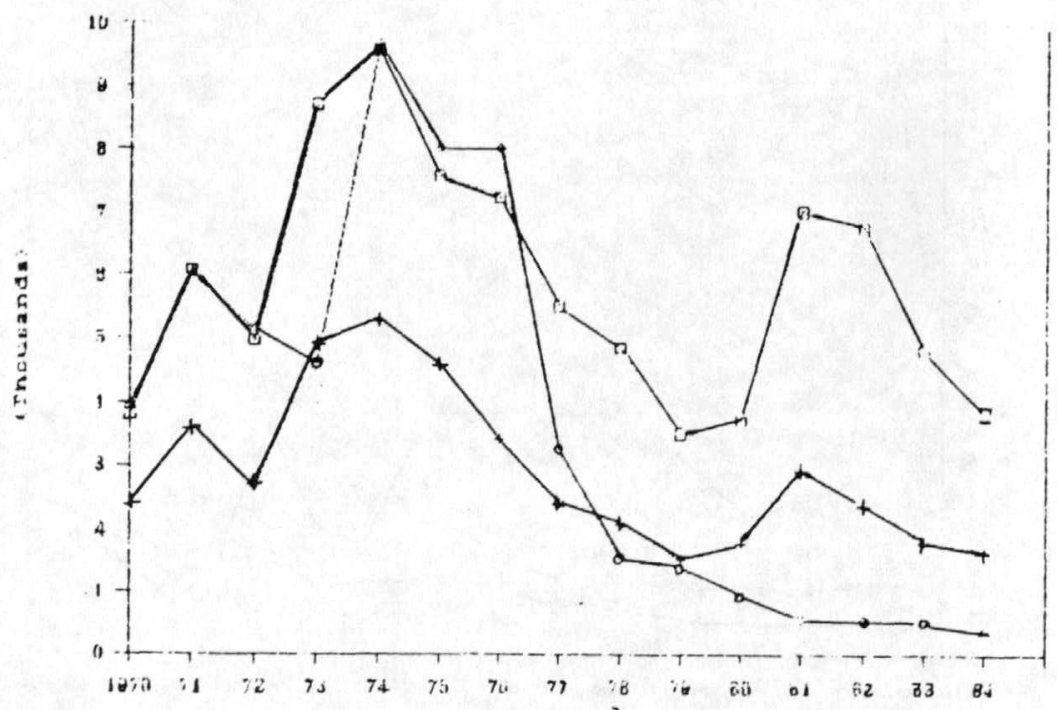

Conflits + Nbre de salariés Nbre de jours 
Graphe 2 : Trends in Number of Employees, Union Membership and Estimaded Unionization Rate

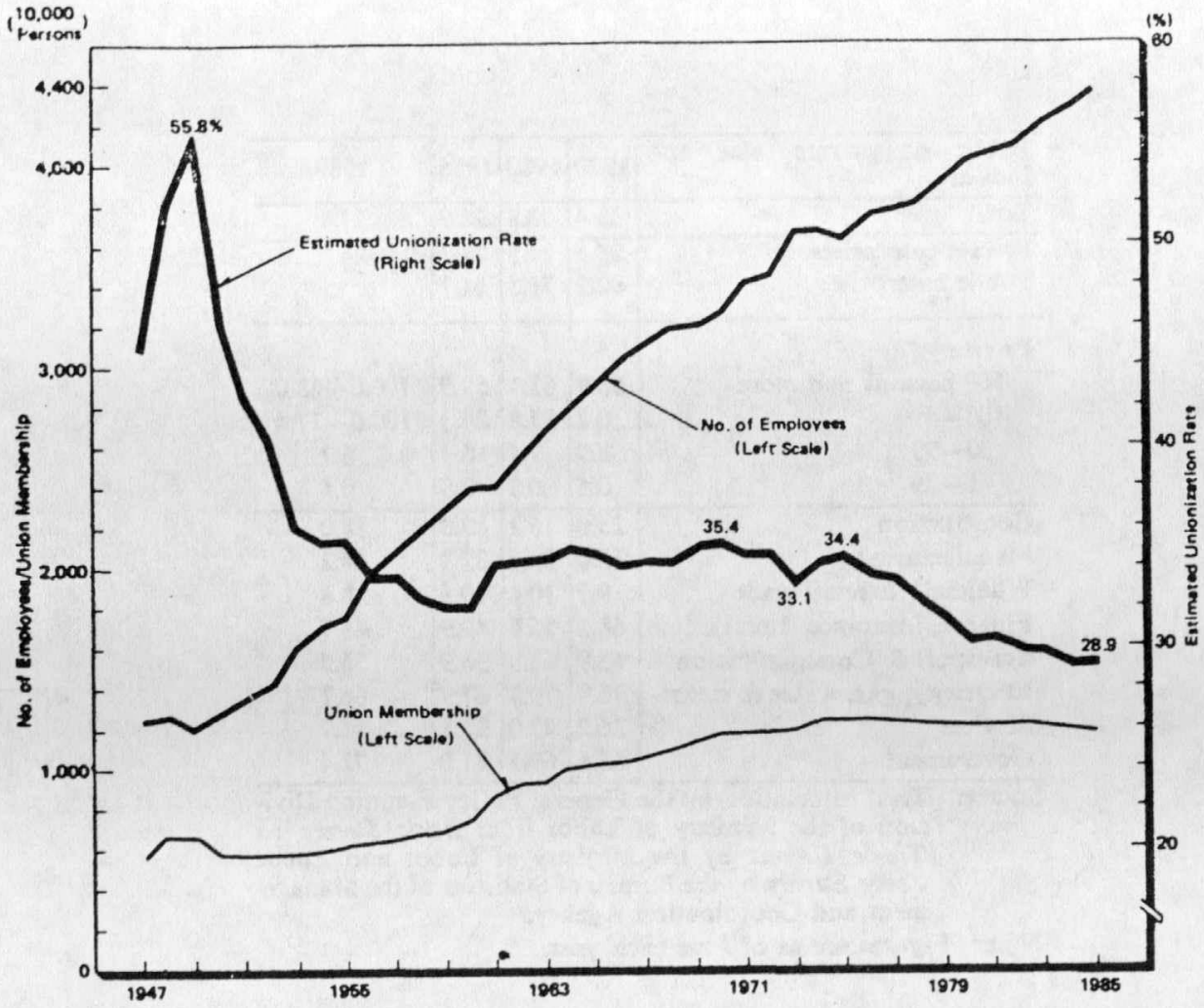

Source : Ministry of Labor, Basic Survey on Trade Unions, 1985. 
Table 1: Trends in the Estimated Unionization Rate by Public or Private, Size and Industry (unit labor unions) (unit : \%)

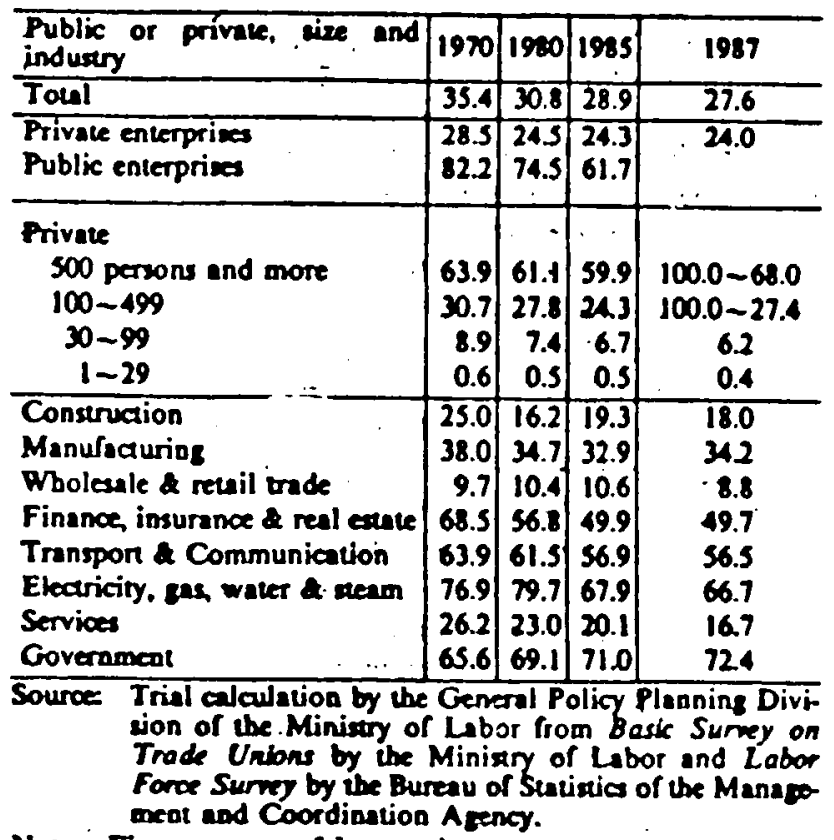

Noce Figures are as of June each year. 
Tableau 2 : Related Indices of Change Of Unionization Rate (unit : \%)

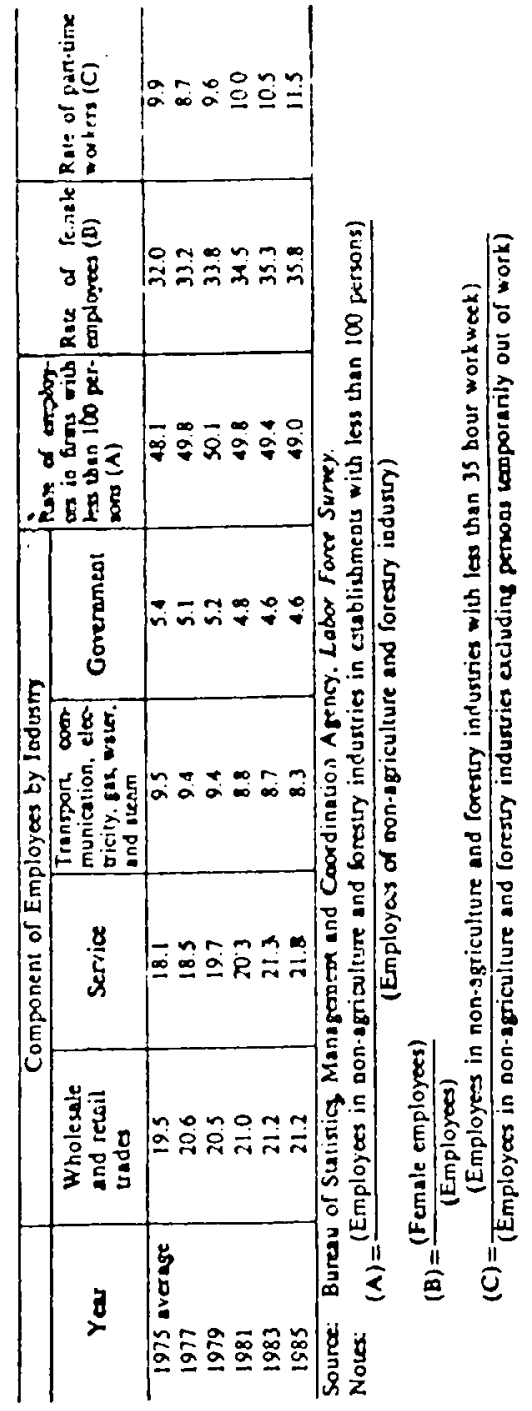


Graphe 3 : International Comparison of Labor's Share in Value Added

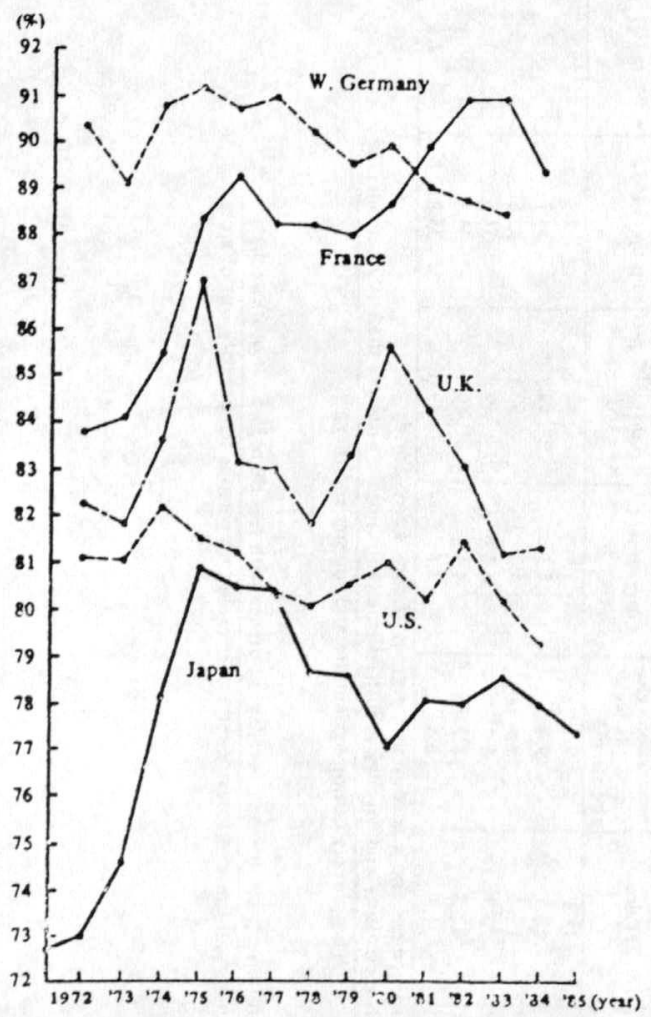

Source : "Economic Growth and Income Distribution", EPA 
Graphe 4 : Trends in Wage Increase in Major Private Firms

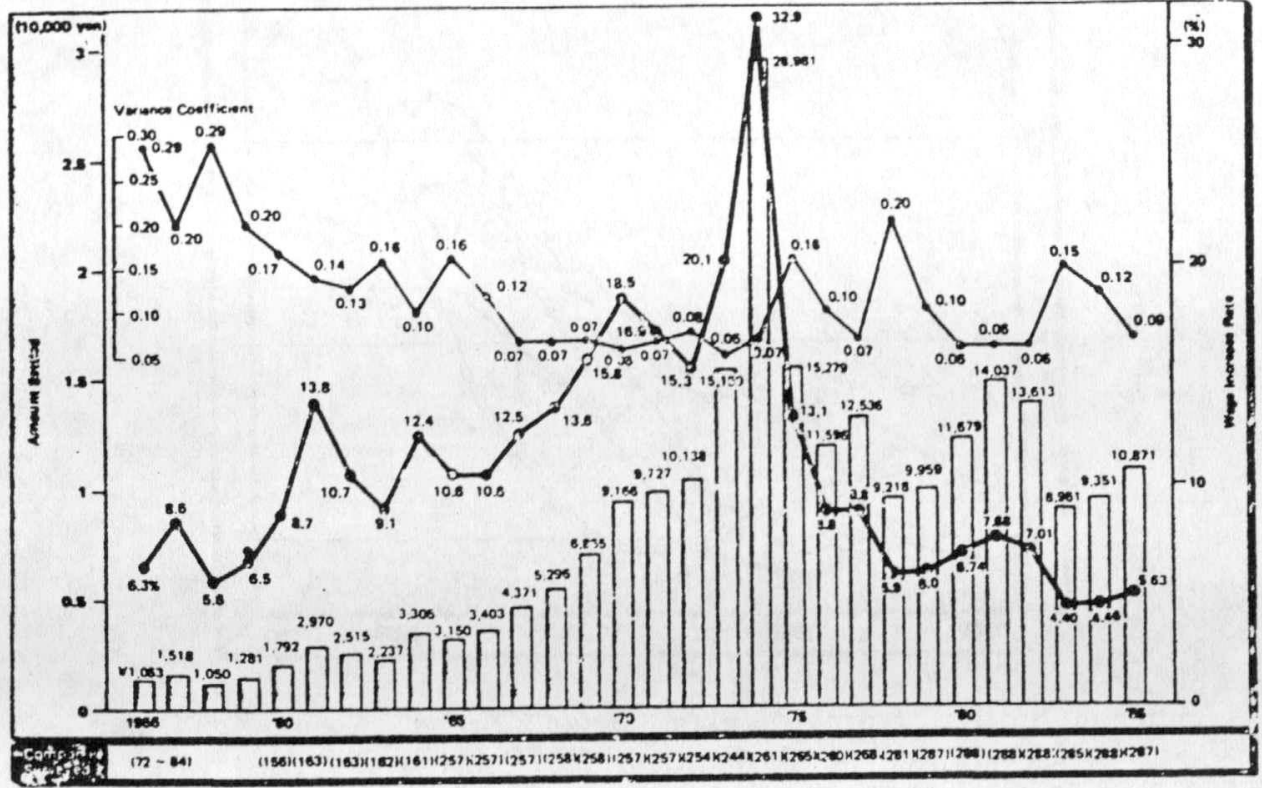

Source : Survey data of the Labor Policy Bureau, Ministry of Labor.

Notes : 1) The amounts settled and the wage increase rates up to 1977 are arithmetical averages, while those in 1978 and thereafter are weighted averages.

2) The variance coefficient is a coefficient of quartiles of the amounts settled in major firms. 
Graphe 5 : Long-term Changes in the Percentage of Old Age Population to the Total Population : An International Comparison

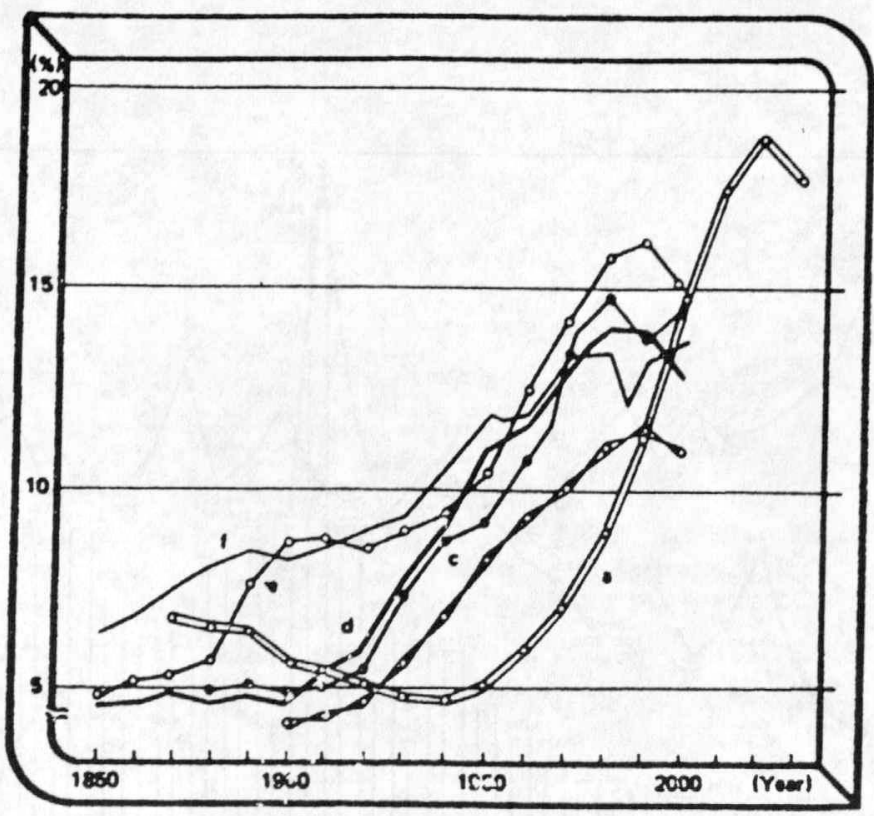

Source: Quoted from Nenkin Seido Kihon Koso Kondenkai (Committee on Basic Ideas for Pension Reforms) Interim Report, 1977.

Notes : Figures correspond to the following countries :
a. Japan
b. U.S.A.
c. West Germany
d. U.K.
e. Sweden
f. France 
Tableau 3 : La composition des parlementaires recommandés par le Rengo

\begin{tabular}{|c|c|c|c|}
\hline & 1)tete & Sénat & Total \\
\hline 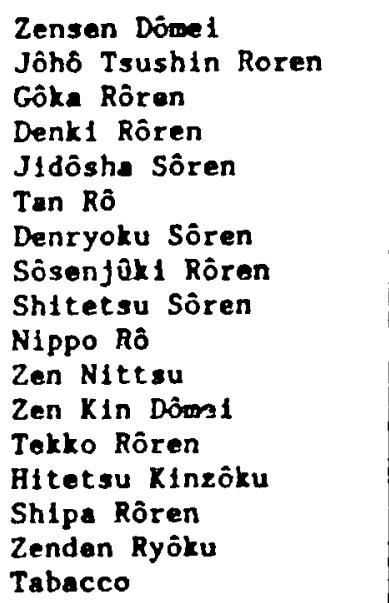 & $\begin{array}{ll}3 & \text { PSD } \\
3 & \text { PSJ } \\
4 & \text { PSJ } \\
2 & \text { PSJ } \\
2 & \text { PSD } \\
3 & \text { PSJ } \\
2 & \text { PSD } \\
2 & \text { PSD } \\
1 & \text { PSJ } \\
2 & \text { PSJ } \\
1 & \text { PSJ } \\
1 & \text { PCD } \\
1 & \text { PSD } \\
1 & \text { PSJ } \\
0 & \\
0 & \\
0 & \end{array}$ & $\begin{array}{ll}2 & \text { PSD } \\
2 & \text { PSJ } \\
0 & \\
2 & \text { PSJ } \\
2 & \text { PSD } \\
1 & \text { PSJ } \\
1 & \text { PSD } \\
1 & \text { PSD } \\
1 & \text { PSJ } \\
0 & \\
1 & \text { PSJ } \\
0 & \\
0 & \\
0 & \\
1 & \text { PSJ } \\
1 & \text { PSJ } \\
1 & \text { PSJ }\end{array}$ & $\begin{array}{l}5 \\
5 \\
4 \\
4 \\
4 \\
4 \\
3 \\
3 \\
2 \\
2 \\
2 \\
1 \\
1 \\
1 \\
1 \\
1 \\
1\end{array}$ \\
\hline Total & 28 & 16 & 44 \\
\hline $\begin{array}{l}\text { Partl socialisto (PSJ) } \\
\text { Partl soclal dépocrate } \\
\text { (PSD) }\end{array}$ & $\begin{array}{l}17 \\
11\end{array}$ & 10 & 27 \\
\hline
\end{tabular}

Source: Rengo NO:4 1987.12.18 
SUMMARY

Japanese trade unionism has undergone some significant changes in the last ten years. The privatisation of the publicly owned railways and telecommunications has led to the weakening of 'sohyo', the most militant union. The least militant organisations have joined in a huge federation -Rengo- which aims to slow down the decline of the trade union movement while rethinking what is at stake in struggles in modern Japanese companies. 